\title{
Pharmacological Approaches to Improve Surgical Outcomes After Retinal Reattachment Surgery
}

\author{
Mark S. BLumenkranz
}

Introduction

Prior to the modern era ushered in by Gonin, with the recognition that retinal detachment is caused by retinal breaks, a variety of nonspecific and pharmacological methods were employed, without predictable success, for the treatment of retinal detachment. It remains true today that the most important pharmacological agent in the diagnosis and treatment of retinal detachment is the mydriatic drop, since without adequate visualization of the fundus and identification of the break, no definitive treatment is possible. Other opportunities still exist for the pharmacological enhancement of retinal reattachment, by improvement in both anatomical and functional results. These include (1) improvement in the final retinal reattachment rate, (2) improvement in visual acuity following successful retinal reattachment, and (3) reduction in complications.

Inasmuch as the majority of routine or uncomplicated retinal detachments can now be accomplished safely by utilizing modern scleral buckling techniques, the risk-benefit ratio of utilizing potentially toxic pharmacological agents under routine circumstances has to be carefully considered in light of the opportunities for marginal potential improvement [1]. However, given the increasing utilization of pneumatic retinopexy, which seems to have a lower initial retinal reattachment rate than conventional scleral buckling [2], the justification for the use of pharmacological agents may be increased. Some authors now propose primary vitrectomy without 
scleral buckling as a first operation, and although the immediate postoperative morbidity may be lower, the final rate of proliferative vitreoretinopathy (PVR) may be higher than scleral buckling alone [3-5]. Similarly, when scleral buckling alone is considered for eyes with early or late forms of proliferative vitreoretinopathy, success rates are considerably lower, and the case for use of an adjuvant agent, either to facilitate intraoperative flattening, such as perfluorocarbon, or one of several different anti-proliferative agents seems better justified [7-9]. Recently, a British group has developed a predictive formula to calculate the risk of PVR following primary retinal reattachment, which may aid in the selection of patients who might benefit most from the use of adjuvant pharmacological methods [10].

\section{Improvement in Visual Acuity}

Improvements in visual acuity following reattachment surgery may be achievable using several different methods. The first would be a reduction in the rate of complications associated with macular dysfunction. These include both macular edema and macular pucker. Both are well-described complications of retinal reattachment surgery as well as other vitreoretinal conditions and may be amenable to pharmacological intervention. Recent reports suggest that intravitreal steroids, principally triamcinolone acetonide, and also dexamethasone in a bioerodable polymer may reduce macular edema associated with several conditions, including diabetic retinopathy, uveitis, and retinal vein occlusion [11, 12]. The use of steroids by other routes of administration, including oral and parenteral, may also be of benefit in preventing macular complications, such as pucker [13].

It may also be possible to improve visual acuity through the enhancement of photoreceptor regeneration in eyes with macula-off retinal detachments. Processes governing photoreceptor renewal and normal alignment of the outer segments following retinal 
reattachment are still poorly understood, and the potential use of pharmacological agents and, specifically, cytokines may be a promising avenue to restoring visual function above and beyond that which might be expected simply by reattachment of the macula, particularly in eyes with more longstanding retinal detachments. To date, there are no well-defined or well-controlled clinical trials that would suggest such an agent is available or on the near horizon, but it is anticipated that in the future this approach may be feasible.

\section{Reduction in Other Non-Macular Complications Limiting Either Anatomic Retinal Reattachment or Normal Physiological Function}

Well-known complications of retinal reattachment surgery include alterations in the intraocular pressure, both glaucoma and hypotony, postoperative inflammation, cataract, motility disorders, and PVR. Pharmacological therapy, both intraoperatively as well as postoperatively, may play a role in the reduction of several of these complications, either singly or in combination. The remainder of this chapter is devoted to a discussion of the principal cause of retinal reattachment failure after primary surgery, PVR, and the methods by which pharmacological therapy might favorably influence this condition.

\section{The Cell Biology of PVR}

PVR is the most common cause of failure following attempted retinal reattachment repair in primary cases, as well as complex forms of retinal detachment. The precise initiating events remain poorly understood, but it is known that the disease is characterized by cell-mediated tractional forces exerted on both preretinal and subretinal membranes, as well as more diffusely to the vitreous gel 
itself $[9,14,15]$. These contractile forces lead to stiffening and elevation of the retina, leakage through retinal breaks, and retinal redetachment typically between 4 weeks and 8 weeks following attempted repair. The condition is more commonly encountered in eyes with large or multiple breaks and is more frequently seen in males and in patients with a history of trauma, hemorrhage, choroidal detachment, or giant retinal tear $[5,10]$. The membranes associated with this condition are composed of a variety of cell types, principally pigment epithelial, glial and myofibroblastic elements, either interspersed within the gel producing compaction of the collagen (hypocellular gel contraction) [15] or defined hypercellular membranes on either the anterior or posterior surface of the retina [14]. Two of the earliest findings in eyes that go on to develop PVR are breakdown of the blood-ocular barriers with increased amounts of intravitreal protein and free-floating dispersed cells, principally retinal pigment epithelium (RPE). The cells appear to enter the growth cycle and actively proliferate in response to chemotactic and mitogenic stimuli and, subsequently, undergo an orderly sequence of steps, including attachment, contraction, and secretion of newly formed extracellular matrix (ECM) [14-21].

Initially, attempts to treat this condition by scleral buckling techniques alone were largely unsuccessful, as were the earliest techniques using vitrectomy without supplemental long-acting tamponades $[6,7,14]$. These initially unsuccessful surgical approaches served as the principal stimulus for a search for antiproliferative agents that might improve success rates. Early studies were performed using triamcinolone acetonide, daunomycin, and fluorouracil in the early 198 os prior to the widespread availability of long-acting gases and silicone oil $[8,22-26]$.

The introduction of long-acting gases, particularly perfluoropropane, and the re-emergence of silicone oil (polydimethylsiloxane) were major steps in the improvement in surgical success rates. The definitive silicone oil study confirmed that while silicone oil was superior to sulfur hexafluoride in providing visual acuity improvement and retinal reattachment in PVR, a companion study 
further amplified the relative equivalence of perfluoropropane to silicone oil in achieving retinal reattachment [27]. Approximately $64-73 \%$ of patients in the latter study achieved complete posterior retinal attachment and $43-45 \%$ achieved functional visual acuity of greater than 5/200 compared with success rates less than half that prior to the utilization of both of these long-acting tamponade techniques [28]. Paradoxically, with the improvement in anatomic reattachment associated with the long-acting tamponades of perfluoropropane and silicone oil, both the medical need and the likelihood of demonstrating a statistically significant treatment benefit by incremental pharmacological methods were reduced, although studies actively continued throughout the 1980s and early 1990 s to develop new pharmacological therapies. In addition to reducing the driving force for development of new drugs due to improved surgical success rates, the use of long-acting tamponades, including silicone oils and long-acting gases, also created unique problems related to bio-availability because of the presence of either a gas-filled eye or an eye filled with an hydrophobic agent [29]. Additionally, subsequent to the publication of the results of the silicone oil study, another pharmaceutical compound used as a temporary intraoperative tamponade, perfluoro- $n$-octane, as well as other liquid perfluorochemicals came into common use. The use of these compounds further improved the success rates for complex forms of retinal detachment repair in conjunction with longacting tamponade even without the use of other pharmacological anti-proliferative agents with success rates reported in the range of $78 \%[30]$.

\section{The General Approach to Pharmacological Therapy}

The search for drugs to inhibit vitreoretinal scarring either preceding or following retinal reattachment surgery has proceeded along lines that target the specific steps of the vitreoretinal scarring response, including cellular activation, proliferation, ECM 
elaboration, and contraction. This process has been facilitated by the use of cell-culture methods for initial screening [31, 32]. Some drugs attack specific points within the cycle, whereas other agents may attack more than one, such as steroids or heparin-like compounds. The various agents have been divided into classes according to their mechanism of action. These include the following: (1) anti-inflammatory agents, (2) drugs that inhibit cellular proliferation, (3) drugs that act on the ECM and cell surface. A general review of these classes of drugs follows.

\section{Anti-Inflammatory Agents}

Corticosteroids were the first agents to be employed in the treatment of experimental PVR and have recently regained currency based upon their widely disparate effects [24]. It is known that steroids exhibit a bimodal effect on cultured fibroblasts, causing stimulation at low doses and inhibition at supraphysiological doses [31]. Triamcinolone acetonide has been shown to reduce experimental PVR in a rabbit model after the injection of cultured fibroblasts [24]. One human clinical study employing oral prednisone showed a reduced rate of macular pucker, a limited form of proliferative response, after retinal reattachment surgery, although it did not affect the ultimate reattachment rate or rate of PVR [13]. Intravitreal steroids, when included in the infusate with heparin, resulted in a lower rate of retinal reoperation in one clinical study [33]. A novel use of intravitreal triamcinolone recently described by Peyman and colleagues involves visualizing remnants of the residual vitreous cortex following injection of a suspension of triamcinolone acetonide and, thereby, enhancing a full removal of cortex and vitreous membranes in a more expeditious manner [34]. 


\section{Drugs That Inhibit Cellular Proliferation}

A variety of subclasses of anti-proliferative agents have been shown to be effective in animal models as well as more recently in clinical trials. The greatest clinical experience has been obtained with fluoropyrimidines [8, 22, 23, 28, 29, 31, 35-42]. Fluoropyrimidines were first chosen because of their potency in inhibiting cellular proliferation in vitro compared with a relative lack of toxicity at high concentrations in toxicological studies [8,31].5-Fluorouracil, the first agent to be tested in detail, has been found to have a median inhibitory dose (ID 50) of between $0.35 \mu \mathrm{g} / \mathrm{ml}$ and $0.71 \mu \mathrm{g} / \mathrm{ml}$ for most ocular and vascular cell types tested [31]. 5-FU has been found to be non-toxic as an intravitreal injection and can be well tolerated both in animals and in humans following intravitreal injections of up to $1.0 \mathrm{mg}$ [8]. The drug is thought to exert its effect by enzymatic conversion into the ribose nucleotide form, which in turn both effects protein synthesis and also the enzyme thymidylate synthetase. Other fluoropyrimidine cogeners, particularly the ribonucleoside (5 FUR), have not only anti-proliferative effects like 5-FU, but also anti-contractile effects, which 5-FU does not seem to exhibit, at least in culture. These, however, are associated with a greater potential toxicity than 5 -FU $[31,32,39]$.

One of the theoretical problems associated with drug therapy employing 5-FU in eyes undergoing surgery for PVR is interaction with the long-acting tamponade, whether it is gas or silicone oil. In one study, a sustained release of a co-drug of 5-FU and fluocinolone pellet was tested in gas-filled eyes and found to be effective in releasing drugs in a manner comparable with non-gas-filled eyes [40, 41]. Co-drugs of 5-FU linked to an alkyl side-chain are soluble in silicone oil, whereas 5-FU itself is not and may be slowly released into the vitreous cavity by hydrolysis of the alkyl side-chain and 5-FU bond. To date, no studies have been performed on humans employing this technique, although it retains some promise [29].

The first randomized prospective clinical trial testing the efficacy of intravitreal 5-FU combined with low-molecular-weight 
heparin was published in eyes undergoing retinal reattachment and thought to be at high risk for the development of PVR rather than having already established PVR. In this study, $200 \mu \mathrm{g} / \mathrm{ml}$ of 5 -FU and $5 \mathrm{IU} / \mathrm{ml}$ of low-molecular-weight heparin were added to the intravitreal infusate in a randomized fashion, with the remainder of the patients receiving a placebo injection into the infusate during vitrectomy. The incidence of post-operative PVR was judged to be lower at 6 months in the treatment group (12.6\%) than the placebo group $(26.4 \%)$, with those in the placebo group also requiring a higher re-operation rate than those in the 5-FU group. However, no differences were seen in the final complication rates [42]. Results of a similar protocol for the treatment of established PVR are still pending at the time of writing of this chapter.

\section{Daunomycin}

Another anti-proliferative agent, daunomycin, has been tested in preclinical and clinical studies. The drug is an anthracycline antibiotic with efficacy in an animal model. Daunomycins appear to have a somewhat lower therapeutic index than 5-FU, principally due to its greater toxicity; but it has been tolerated in animals and in humans as a continuous intravitreal infusion of $7.5 \mu \mathrm{g} / \mathrm{ml}$ for $10 \mathrm{~min}$. In pilot studies of patients undergoing vitrectomy, daunomycin was felt to be effective $[26,43]$. A variety of other agents have been employed for the treatment of experimental PVR, with no significant published data yet in human trials. These include retinoids, which play an important role in the differentiation and proliferation of various cell types, including RPE. The treatment of RPE cells with vitamin A (all-trans-retinol) significantly inhibits cellular proliferation migration in vitro as well as having effects on morphology [44]. Immunotoxins composed of a monoclonal antibody linked to a biological toxin have been employed in experimental models, including an antibody against the human transferrin receptors to the A chain of ricin [45]. 
Another chemotherapeutic agent useful in cancer and also in the treatment of coronary restenosis, Taxol, has been tested for efficacy in experimental models in the eye. It appears to act as a promoter rather than an inhibitor of microtubular assembly and inhibits cell-mediated contraction of a collagen gel as well as experimental retinal detachment in various animal models [46]. A related cytoskeletal agent, Colchicine, also inhibits RPE astrocyte and fibroblast proliferation in addition to migration and was shown in one animal model to have some beneficial effects on PVR, although it has not yet been proven to be beneficial in any human studies [47].

\section{Drugs Acting on the ECM}

Drugs which act on the interface between cells and the ECM have the potential to inhibit intraretinal scarring at a relatively earlier step than simple proliferation. Heparin and related peptides have a multitude of effects on cells and their interaction with the ECM. Heparin is a glycosaminoglycan derived from heparin sulfate, which binds to several ECM proteins, including fibronectin, laminin, and vitronectin [48]. In addition to its antithrombotic properties, for which it was first discovered and processed, heparin clearly has important effects on a variety of growth factors. It actively binds fibroblast growth factor, platelet-derived growth factor, and endothelial-cell growth factor. Soluble heparin causes an increase in cell spreading and produces changes in the cytoskeleton of smooth muscles. It also inhibits the polymerization of type-1 collagen and reduces cell-mediated contraction of collagen gels when cultured fibroblasts or RPE cells are interspersed within a collagen matrix. This may be a process analogous to hypocellular gel contraction, an important attack point in the prevention of PVR $[15,48]$.

Because heparins have significant anticoagulant affects, they may result in hemorrhagic complications, and this stimulated the 
search for compounds with heparin-like qualities on growth factors, but without the potential hemorrhagic issues. It is known that fractionation of longer chains of heparin into smaller molecular weight fragments causes loss of some of the anticoagulant activity while preserving the ECM effects. As a result, low-molecular-weight fractions of heparin with a molecular weight of 5000 or less retain their ability to catalyze the inhibition of Factor Xa, but lose their ability to directly inhibit thrombin [49]. When low-molecularweight heparin was introduced into the infusate during vitrectomy in an animal model, fibrin formation was markedly reduced without any coincident increase in intraocular hemorrhage [50]. Use of $5 \mathrm{IU} / \mathrm{ml}$ of low-molecular-weight heparin in the infusate during creation of an experimental model of proliferative vitreoretinopathy reduced the rate of traction detachment from $77 \%$ to $28 \%$ at 3 months [51]. These encouraging results led to the inclusion of lowmolecular-weight heparin in the infusate, along with 5-FU, which was studied in the prevention of PVR in humans [15, 42, 50, 51].

In another important PVR trial, when conventional heparin (1 IU/ml) was combined with a steroid dexamethasone $(5 \mathrm{mg} / \mathrm{ml})$ in the infusate, there was a slight increase in the retinal reattachment rate compared with controls from $65 \%$ to $80 \%$ in addition to a reduction in the rate of reproliferation from $26.5 \%$ to $16 \%$. A mild increase in the rate of hyphema and vitreous hemorrhage was seen, although it was not judged to be clinically significant [33].

\section{Summary}

Pharmacological methods remain a promising potential adjunct to the successful treatment of retinal detachment. In addition to conventional strategies, including adequate pupillary dilatation, control of inflammation by anti-inflammatory agents, and intraocular pressure by ocular hypotensive agents, drugs may play a further role by inhibiting other late complications. These include proliferation and macular edema. Steroids, fluoropyrimidines, and he- 
parin-like compounds, particularly low-molecular-weight heparin, all appear to be potentially useful agents either singly, or in combination. The ability to combine agents together with differing mechanisms of action, either as a single intravitreal injection, or, more recently, as a component of the infusate or with extended delivery devices, opens up new therapeutic avenues. Issues related to bioavailability, particularly in eyes with long-acting tamponades, either gas or silicone oil, are particularly challenging but intriguing. The use of modern bioerodable polymers and other fixed extended delivery devices may further enhance the utilization of such agents. In the future, additional capabilities, including the use of intravitreal steroids for control of macular edema and cytokines for the improvement of photoreceptor recovery, may further improve visual acuity results beyond those that might be expected when anatomic reattachment reaches a high plateau. Further understanding of the cellular biology of retinal reattachment and macular function will undoubtedly lead to concomitant improvements in therapeutic advances.

\section{References}

1. Lincoff H, Kreissig I (1972) The treatment of retinal detachment without drainage of subretinal fluid. Trans Am Acad of Ophthalmol Otolaryngol 76:1221-1232

2. Han DP, Mohsin NC, Guse CE, Harz A, Tarkanian CN (1999) Comparison of pneumatic retinopexy and scleral buckling in the management of primary rhegmatogenous retinal detachment. Southern Wisconsin Pneumatic Retinopexy Study Group. Am J Ophthalmol 127: 741-743

3. Campo RV, Sipperley JO, Sneed SR, Park DW et al (1999) Pars plana vitrectomy without scleral buckle for pseudophakic retinal detachments. Ophthalmology 106:1811-1815

4. Brazitikos PD, Androudi S, D’Amico DJ, Papadopoulis N et al (2003) Perfluorocarbon liquid utilization in primary vitrectomy repair of retinal detachment with multiple breaks. Retina 23:615-621 
5. Cowley M, Conway B, Campochiaro P, Kaiser D, Gaskin H (1989) Clinical risk factors for proliferative vitreoretinopathy. Arch Ophthalmol 107:1147-1151

6. Grizzard WS, Hilton GF (1982) Scleral buckling for retinal detachments complicated by periretinal proliferation. Arch Ophthalmol 100: 419-422

7. Sternberg P Jr, Machemer R (1985) Results of conventional vitreous surgery for proliferative vitreoretinopathy. Am J Ophthalmol 100: 141-146

8. Blumenkranz M, Hernandez E, Ophir A, Norton EWD (1984) 5-Fluorouracil: new applications in complicated retinal detachment for an established antimetabolite. Ophthalmology 91:122130

9. Blumenkranz MS (1988) Management of complicated retinal detachment. In: Tso MOM (ed) Retinal diseases: biomedical foundations and clinical management. JB Lippincott, Philadelphia

10. Asaria $\mathrm{RH}$, Kon $\mathrm{CH}$, Bunce $\mathrm{C}$, Charteris DC et al (2001) How to predict proliferative vitreoretinopathy: a prospective study. Ophthalmology 108:1184-1186

11. Martidis A, Duker JS, Greenberg PB, Rogers AH et al (2002) Intravitreal triamcinolone for refractory diabetic macular edema. Ophthalmology 109:920-927

12. Greenberg PB, Martidis A, Rogers AH, Duker JS, Reichel E (2002) Intravitreal triamcinolone acetonide for macular oedema due to central retinal vein occlusion. Br J Ophthalmol 86:247-248

13. Koerner F, Merz A, Gloor B, Wagner E (1982) Postoperative retinal fibrosis - a controlled clinical study of systemic steroid therapy. Graefes Arch Clin Exp Ophthalmol 219:268-271

14. Machemer R (1977) Massive periretinal proliferation: a logical approach to therapy. Trans Am Ophthalmol Soc 75:556-586

15. Blumenkranz MS, Hartzer MK (2000) The mechanism of action of drugs for the treatment of vitreoretinal scarring. In: Retina, vol. 3. Ryan S, Glaser B (eds) CV Mosby, St. Louis, pp 2281-2293

16. Campochiaro PA, Bryan JA III, Conway BP, Jaccoma EH (1986) Intravitreal chemotactic and mitogenic activity implications of bloodretinal barrier breakdown. Arch Ophthalmol 104:1685-1687

17. Campochiaro PA, Glaser BM (1986) Mechanisms involved in retinal pigment epithelial cell chemotaxis. Arch Ophthalmol 104: 277-280 
18. Yeo JH, Sadeghi J, Campochiaro PA, Green WR, Glaser BM (1986) Intravitreous fibronectin and platelet-derived growth factor: new model for tractional retinal detachment. Arch Ophthalmol 104:417-421

19. Verdoorn C, Renardel de Lavalette VW, Dalma-Weizhaus J, Orr GM, Sorgente N, Ryan SJ (1986) Cellular migration, proliferation and contraction: an in vitro approach to a clinical problem - proliferative vitreoretinopathy. Arch Ophthalmol 104:1216-1219

20. Vidaurri-Leal J, Glaser B (1984) Effect of fibrin on morphologic characteristics of retinal pigment epithelial cells. Arch Ophthalmol 102:1376-1379

21. Vidaurri-Leal J, Hohman R, and Glaser BM (1984) Effect of vitreous on morphologic characteristics of retinal pigment epithelial cells: a new approach to the study of proliferative vitreoretinopathy. Arch Ophthalmol 102:1220-1223

22. Blumenkranz MS, Ophir A, Claflin A, Hajek AS (1982) Fluorouracil for the treatment of massive periretinal proliferation. Am J Ophthalmol 94:458-467

23. Stern WH, Lewis GP, Erickson PA, Guerin CJ, Anderson DH, Fisher SK, O’Donnell JJ (1983) Fluorouracil therapy for proliferative vitreoretinopathy after vitrectomy. Am J Ophthalmol 96:33-42

24. Tano Y, Sugita G, Abrams G, Machemer R (1980) Inhibition of intraocular proliferations with intravitreal corticosteroids. Am J Ophthalmol 89:131-136

25. Van Bockxmeer FM, Martin CE, Constable IJ (1985) Models for assessing scar tissue inhibitors. Retina 5:47-60

26. Wiedemann P, Lemmen K, Schmiedl R, Heimann K (1987) Intraocular daunorubicin for the treatment and prophylaxis of traumatic proliferative vitreoretinopathy. Am J Ophthalmol 104:10-14

27. Anonymous (1992) Vitrectomy with silicone oil or sulfur hexafluoride gas in eyes with severe proliferative vitreoretinopathy: results of a randomized clinical trial. Silicone Study Report 1. Arch Ophthalmol 110:770-779

28. Anonymous (1992) Vitrectomy with silicone oil or perfluoropropane gas in eyes with severe proliferative vitreoretinopathy: results of a randomized clinical trial. Silicone Study Report 2. Arch Ophthalmol 110:780-792

29. Jolimaitre P, Malet-Martino M, Martino R (2003) Fluorouracil prodrugs for the treatment of proliferative vitreoretinopathy: formulation in silicone oil and in vitro release fluorouracil. Int J Pharm 259:181-192 
30. Scott IU, Flynn HW Jr, Murray TG, Feuer WJ (2003) Perfluoron study group. Outcomes of surgery for retinal detachment associated with proliferative vitreoretinopathy using perfluoro-n-octane: a multicenter study. Am J Ophthalmol 136:454-463

31. Blumenkranz MS, Claflin A, Hajek AS (1984) Selection of therapeutic agents for intraocular proliferative disease: cell culture evaluation. Arch Ophthalmol 102:598-604

32. Blumenkranz MS, Hartzer MK, Hajek AS (1987) Selection of therapeutic agents for intraocular proliferative disease. II. Differing antiproliferative activity of the fluoropyrimidines. Arch Ophthalmol 105:396-399

33. Williams RG, Chang S, Comaratta M, Simoni G (1996) Does the presence of heparin and dexamethasone in the vitrectomy infusate reduce reproliferation in proliferative vitreoretinopathy? Graefes Arch Clin Exp Ophthalmol 234: 496-503

34. Peyman GA, Cheema R, Conway MD, Fang T (2000) Triamcinolone acetonide as an aid to visualization of the vitreous and the posterior hyaloid during pars plana vitrectomy. Retina 20:554-555

35. Barrada A, Peyman GA, Case J, Fishman G, Thomas A, Fiscella R (1984) Evaluation of intravitreal 5-fluorouracil, vincristine, VP16, doxorubicin, and thiotepa in primate eyes. Ophthalmic Surg 15:767-769

36. Berger AS, Cheng CK, Pearson A, Ashton P, Crooks P, Cynkowski T, Cynkowska G, Jaffe GJ (1996) Intravitreal sustained release corticosteroid-5-fluorouracil conjugate in the treatment of experimental proliferative vitreoretinopathy. Invest Ophthalmol Vis Sci 37:23182325

37. Rubsamen PE, Davis P, Hernandez E, O'Grady G, Cousins SW (1994) Prevention of experimental proliferative vitreoretinopathy with a biodegradable intravitreal implant for the sustained release of fluorouracil. Arch Ophthalmol 112: 407-413

38. Stern WH, Guerin CJ, Erickson PA, Lewis GP, Anderson DH, and Fisher SK (1983) Ocular toxicity of fluorouracil after vitrectomy. Am J Ophthalmol 96: 43-51

39. Hartzer MK, Blumenkranz MS, Hajek AS, Dailey WD, Cheng M, Margherio AS (1989) Selection of therapeutic agents for the treatment of intraocular proliferative disease. Part 3. Effects of fluoropyrimidines on cell-mediated contraction. Exp Eye Res 48:321-328

40. Berger AS, Cheng CK, Pearson A, Ashton P, Crooks P, Cynkowski T, Cynkowska G, Jaffe GJ (1996) Intravitreal sustained release cortico- 
steroid-5-fluorouracil conjugate in the treatment of experimental proliferative vitreoretinopathy. Invest Ophthalmol Vis Sci 37:2318-2325

41. Perkins SL, Galemore R, Yang CH, Guo H, Ashton P, Jaffe GJ (2000) Pharmacokinetics of the flucinolone/5-fluorouracil codrug in the gasfilled eye. Retina 20:514-519

42. Asaria RH, Kon CH, Bunce C, Charteris D, Wong D, Khaw P, Aylward G (2001) Adjuvant 5-fluorouracil and heparin prevents proliferative vitreoretinopathy: results from a randomized, double-blind, controlled clinical trial. Ophthalmology 108:1179-1183

43. Wiedemann P, Leinung C, Hilgers RD, Heimann K (1991) Daunomycin and silicone oil for the treatment of proliferative vitreoretinopathy. Graefes Arch Clin Exp Ophthalmol 229:150-152

44. Campochiaro PA, Hackett SF, Conway BP (1991) Retinoic acid promotes density-dependent growth arrest in human retinal pigment epithelial cells. Invest Ophthalmol Vis Sci 32:65-72

45. Mandava N, Blackburn P, Paul D, Wilson M, Read S, Alspaugh E, Tritz R, Barber J, Robbins J, Kruse C (2002) Ribozyme to proliferating cell nuclear antigen to treat proliferative vitreoretinopathy. Invest Ophthalmol Vis Sci 43:3338-3348

46. Van Bockxmeer FM, Martin CE, Thompson DE, Constable IJ (1985) Taxol for the treatment of proliferative vitreoretinopathy. Invest Ophthalmol Vis Sci 26: 1140-1147

47. Lemor M, Yeo JH, Slater B (1986) Oral colchicine for the treatment of experimental retinal detachment. Arch Ophthalmol 104:1226-1229

48. Blumenkranz M, Hartzer M, Iverson D (1992) An overview of potential applications of heparin in vitreoretinal surgery. Retina 12[Suppl]: $\mathrm{S}_{71-S 74}$

49. Bratt G, Tornebohm E, Lockner D, Bergstrom K (1985) A human pharmacological study comparing conventional heparin and a low molecular weight heparin fragment. Thromb Haemost 53:208-211

50. Iverson DA, Katsura H, Hartzer MK, Blumenkranz MS (1991) Inhibition of intraocular fibrin formation following infusion of lowmolecular weight heparin during vitrectomy. Arch Ophthalmol Vis Sci 109:405-409

51. Chapman C, Iverson D, Hartzer M, Minelli E, and Blumenkranz M (1992) The effect of low molecular weight heparin on proliferative vitreoretinopathy induced in the rabbit eye. Invest Ophthalmol Vis Sci 33[Suppl]:818 